\title{
Optimal Sensor Placement For Fuel Cell System Diagnosis using BILP Formulation
}

\author{
Fatiha Nejjari, Ramon Sarrate and Albert Rosich
}

\begin{abstract}
This paper presents the application of a new methodology for Fault Detection and Isolation (FDI) to a Fuel Cell System. The work is devoted to find an optimal set of sensors for model-based FDI. The novelty is that binary integer linear programming (BILP) is used in the optimization formulation, leading to a reformulation of the detectability and isolability specifications as linear inequality constraints. The approach has been successfully applied to a Fuel Cell System.
\end{abstract}

\section{INTRODUCTION}

In model-based FDI (Fault Detection and Isolation), faults are modeled as deviations of parameter values or unknown signals, and diagnostic models are often brought back to a residual form. The main approaches to construct residuals are based on using Analytical Redundancy Relations (ARRs) generated either using the parity space [1] or observer approaches [2]. In [3] the sensor placement problem is solved by the analysis of a set of possible ARRs using algorithms of cycle generation in graphs. Some other results devoted to sensor placement for diagnosis using graph tools can be found in [4], [5], [6], [7], [8], [9] and [10]. All these works use a structural model-based approach and define different diagnosis specifications to solve the sensor placement problem.

A structural model is a coarse model description, based on a bi-partite graph, that can be obtained early in the development process, without major engineering efforts. This kind of models is suitable to handle large scale systems since efficient graph-based tools can be used and does not have numerical problems. However, only best case results are obtained. More information about structural modeling applied to fault diagnosis can be found in [11].

In [12], an optimal sensor placement for model-based FDI requires finding the set of all possible ARRs, considering that all possible candidate sensors are installed. Then, a set of sensors that minimizes the total cost of the network is selected such that the resulting ARRs satisfy that a pre-established set of faults can be detected and isolated. The optimization problem is casted as a Binary Integer Non Linear Programming (BINLP) problem [13], where the optimization vector states whether a sensor is installed or not and FDI specifications are translated into constraints. However, the non-linear nature of such constraints lead to a high computational complexity of the resulting optimization problem.

This work was supported by CICYT (ref. DPI-2008-01996) of Spanish Ministry of Education

All authors are with the Automatic Control Department, Universitat Politècnica de Catalunya, Rambla de Sant Nebridi, 10, 08222 Terrassa, Spain fatiha.nejjarieupc.edu
An alternative BINLP sensor location for diagnosis is proposed in [9]. This formulation does not require finding the set of all possible ARRs. However, it is only applicable to linear structured systems [14].

In [15], the approach in [12] is enhanced by formulating a Binary Integer Linear Programming (BILP) problem. The FDI specifications are formulated as linear constraints and the objective cost function is also linear, so that the BILP problem can be efficiently solved by an LP-based branchand-bound algorithm.

This paper is an extension of the work presented in [15]. In order to demonstrate the efficiency of this optimal sensor placement formulation a complex non-linear system is chosen as a case study.

Fuel cell systems are receiving much attention in the last decade as good candidates for clean electricity generation. Here, a fuel cell system benchmark is used and some faults are defined to be diagnosed. The fuel cell system model is a complex model which involves a wide range of nonlinear equations (lookup tables, piecewise functions, saturations, non-linear dynamic equations, etc.), and moreover the operating point may also change. So, a model linearization approach is not feasible.

The paper is organized as follows: In Section II, sensor placement is formulated as a BILP problem. The FDI specifications are formulated as binary linear constraints in Section III. Section IV summarizes the BILP formulation of the sensor placement problem and extends it to also include ARRs optimal selection. Section V describes the application of the FDI BILP formulation to a Fuel Cell System. Finally, some conclusions and remarks are given in Section VI.

\section{SENSOR PLACEMENT FOR FDI}

A standard optimization problem using BILP can be formulated as a linear objective function and constrained by linear inequality constraints:

$$
\begin{aligned}
\min _{\mathbf{x}} \mathbf{c}^{T} \mathbf{x} \text { subject to: } & \\
& \mathbf{A x} \leq \mathbf{b} \\
& \mathbf{x} \text { is binary }
\end{aligned}
$$

The main constraint is that any element of the optimization vector $\mathbf{x}$ must be binary, i.e. $\forall x \in \mathbf{x}: x \in\{0,1\}$. Furthermore, matrix $\mathbf{A}$ and vector $\mathbf{b}$ form the linear inequality constraints. Finally, $\mathbf{c}$ is a cost vector of the linear objective function.

In this case the sensor placement problem for FDI can be defined as finding a minimal set of sensors to be installed in the system such that faults can be detected and isolated. 
The sensor placement problem can be formulated as a BILP problem where the set of candidate sensors to be installed is represented by the optimization variable vector. This means that if the entry $x \in \mathbf{x}$ equals 1 , the corresponding sensor must be installed whereas if $x$ equals 0 , the sensor does not need to be installed.

Furthermore, by means of the $\mathbf{c}$ vector a cost can be assigned to each sensor in order to find an optimal solution based in some criterion, e.g. minimal cardinality, minimal economical price, etc.

This approach requires to formulate all constraints as linear inequalities. Thus, for sensor placement for FDI, fault detectability and fault isolability constraints must be expressed as in (2).

\section{CONSTRAintS FORMUlATION}

In model-based FDI, ARRs are used to check the consistency between the model and system measurements. An ARR can be obtained from a subset of model equations by eliminating unknown variables through the convenient manipulation of the equations. Therefore, an ARR is an expression that only depends on known (measured) variables. Structural analysis theory has been extensively used in model-based FDI to generate the ARRs from the model equations [11], [8], [16].

It is straightforward to establish a relation between the ARRs set and the set of known variables. This relation is represented by a bi-adjacency matrix where the row set is the ARRs set and the column set is the sensors set. Let $n$ be the number of ARRs and $k$ be the number of candidate sensors, then the biadjacency matrix $\mathbf{M}=\left[m_{i j}\right]$ is a $n \times k$ matrix defined by

$$
m_{i j}= \begin{cases}1 & \text { if ARR } i \text { depends on sensor } j \\ 0 & \text { otherwise }\end{cases}
$$

Knowing which equations are related to a certain ARR it is possible to determine the set of faults that an ARR is sensitive to. This ARR-fault relation is known in the literature by the Fault Signature Matrix (FSM) [11]. Let $l$ be the number of system faults (i.e. faults that affect to system components other than sensors) to be diagnosed, then the biadjacency matrix $\mathbf{F}=\left[f_{i j}\right]$ is a $n \times l$ matrix defined by

$$
f_{i j}= \begin{cases}1 & \text { if fault } j \text { may affect ARR } i \\ 0 & \text { otherwise }\end{cases}
$$

Remark that, the FSM $F$ will refer to system faults. A similar FSM will be considered for sensor faults, which will be denoted by $\mathbf{F}_{\mathbf{q}}=\left[f_{q_{i j}}\right]$ and easily deduced from $\mathbf{M}$ as $\mathbf{F}_{\mathbf{q}}=\mathbf{M}$.

In the following sections, the constraint formulation proposed in [15] will be revisited.

\section{A. ARR selector constraint}

Given a subset of installed sensors there may be some ARRs that are not valid since they depend on candidate sensors not chosen for installation. Let $\mathbf{q}=\left[q_{1}, \cdots, q_{k}\right]^{T}$ be the binary vector that denotes whether a sensor is installed. Then, an ARR $i$ is called non-selectable if there is a sensor $j$ such that $q_{j}=0$ and $m_{i j}=1$. This motivates the $A R R$ selector:

$$
\rho_{i}=\prod_{j=1}^{k}\left[m_{i j} q_{j}+\left(1-m_{i j}\right)\right]
$$

Note that $\rho_{i}$ is a binary variable such that if ARR $i$ is non-selectable then $\rho_{i}$ equals 0 . However, expression (6) is non-linear and can not be casted as a constraint in (2). In order to do so, remark that inequality (7) holds as long as ARR $i$ is non-selectable.

$$
\sum_{j=1}^{k}\left[m_{i j} q_{j}+\left(1-m_{i j}\right)\right]<k
$$

Next, introducing the binary variable $\rho_{i}$ in inequality (7), the expression (8) is obtained, which is equivalent to the $A R R$ selector in (6).

$$
\sum_{j=1}^{k}\left[m_{i j} q_{j}+\left(1-m_{i j}\right)\right]-k \rho_{i} \geq 0
$$

Note however that expression in (8) implies that

$$
\text { ARR } i \text { is not valid } \rightarrow \rho_{i}=0
$$

whereas the reverse is not true. This means that $\rho_{i}$ can be viewed as a dummy variable in the optimization problem. This variable is forced to zero as long as the corresponding ARR is non-selectable. Otherwise, an ARR may be chosen by the optimization algorithm (i.e., setting $\rho_{i}$ to 1 ) depending on the other FDI specifications.

Now, equation (8) is linear. Therefore, it is suitable for BILP formulation. Rewriting (8) in vector form and extending it for all the $n$ ARRs, we have

$$
\left[\begin{array}{ccc}
m_{i 1} & \cdots & m_{i k} \\
\vdots & \ddots & \vdots \\
m_{n 1} & \cdots & m_{n k}
\end{array}\right]\left[\begin{array}{c}
q_{1} \\
\vdots \\
q_{k}
\end{array}\right]-k\left[\begin{array}{c}
\rho_{1} \\
\vdots \\
\rho_{n}
\end{array}\right]+\left[\begin{array}{c}
\beta_{1} \\
\vdots \\
\beta_{n}
\end{array}\right] \geq \mathbf{0}_{n \times 1}
$$

where $\beta_{i}=\sum_{j=1}^{k}\left(1-m_{i j}\right)$ for $i=\{1, \cdots, n\}$. Equation (9) can be written in a compact form as

$$
\left[\begin{array}{ll}
-\mathbf{M} & k \mathbf{I}_{n}
\end{array}\right]\left[\begin{array}{l}
\mathbf{q} \\
\rho
\end{array}\right] \leq \beta
$$

where variables vector $\rho=\left[\begin{array}{lll}\rho_{1} & \cdots & \rho_{n}\end{array}\right]^{T}$ is the set of ARR selectors and $\beta=\left[\begin{array}{lll}\beta_{1} & \cdots & \beta n\end{array}\right]^{T}$ is a vector of coefficients.

Expression (10) has the same form as (2). Furthermore, the optimization variable vector is augmented by including the ARR selector, i.e. $\mathbf{x}=\left[\begin{array}{ll}\mathbf{q}^{T} & \rho^{T}\end{array}\right]^{T}$. 


\section{B. Fault detectability constraint}

A fault is structurally detectable if there exists at least one ARR that can be affected by this fault. Hence, the $A R R$ selector plays an important role since all non-selectable ARR must be rejected from the detectability study.

Since system and sensor faults are considered in this paper, the number of equations needed to check fault detectability is $l+k$. Next, both type of constraints are deduced.

1) System faults detectability:

Given a fault $j$, the following expression holds:

$$
\text { a system fault } j \text { is detectable } \leftrightarrow \sum_{i=1}^{n}\left(f_{i j} \rho_{i}\right) \geq 1
$$

Equation (11) can be extended to all system faults, and written in compact form as

$$
\left[\begin{array}{ll}
\mathbf{0}_{l \times k} & -\mathbf{F}^{T}
\end{array}\right]\left[\begin{array}{c}
\mathbf{q} \\
\rho
\end{array}\right] \leq-\mathbf{1}_{l \times 1}
$$

Therefore, the set of system faults is detectable if constraint (12) holds.

2) Sensor faults detectability:

A similar expression to (11) is used for sensor fault detectability:

$$
\text { a sensor fault } j \text { is detectable } \leftrightarrow \sum_{i=1}^{n}\left(f_{q_{i j}} \rho_{i}\right) \geq q_{j}
$$

Note that for a non-installed sensor, $q_{j}=0$ and inequality (13) always holds, meaning that no detectability property is expected for this sensor fault. However, as long as a sensor is chosen for installation $\left(q_{j}=1\right)$, equation (13) becomes equivalent to (11).

Equation (13) can be extended to all sensor faults, and written in compact form as

$$
\left[\begin{array}{ll}
\mathbf{I}_{k} & -\mathbf{F}_{\mathbf{q}}^{T}
\end{array}\right]\left[\begin{array}{l}
\mathbf{q} \\
\rho
\end{array}\right] \leq \mathbf{0}_{k \times 1}
$$

\section{Fault isolability constraint}

Two faults are structurally isolable if their corresponding signatures in the FSM are different. This is true as long as ARR-based exoneration is assumed [8].

Since system and sensor faults are considered in this paper, the number of equations needed to check fault isolability is the 2-combination of $l+k$ faults, $C_{2}^{l+k}$. Next, three types of constraints are deduced depending on whether system or sensor faults are considered.

1) Fault isolability between system faults:

Given two system faults $j_{1}$ and $j_{2}$, inequality (15) holds as long as their signatures in the FSM are different.

$$
j_{1} \text { and } j_{2} \text { are isolable } \leftrightarrow \sum_{i=1}^{n}\left|f_{i j_{1}}-f_{i j_{2}}\right| \rho_{i} \geq 1
$$

Equation (15) can be extended to any combination of two system faults, and written in compact form as:

$$
\left[\begin{array}{ll}
\mathbf{0}_{C_{2}^{l} \times k} & -\mathbf{F}_{\mathbf{I}_{1}}{ }^{T}
\end{array}\right]\left[\begin{array}{l}
\mathbf{q} \\
\rho
\end{array}\right] \leq-\mathbf{1}_{C_{2}^{l} \times 1}
$$

where $\mathbf{F}_{\mathbf{I}_{\mathbf{1}}}=\left[f_{I_{1 \text { im }}}\right]$ is a $n \times C_{2}^{l}$ matrix with:

$$
f_{I_{1 i m}}=\left|f_{i j_{1}}-f_{i j_{2}}\right| \quad \forall j_{1}, j_{2} \in\{1, \ldots, l\}: j_{1}<j_{2}
$$

where $m$ indexes in lexicographical order ${ }^{1}$ the $C_{2}^{l}$ system faults combinations.

2) Fault isolability between system faults and sensor faults:

Isolability involving a sensor fault depends on whether the corresponding sensor is considered for installation or not. So, the condition for isolability between a system fault and a sensor fault can be stated as:

$$
\begin{gathered}
\text { a system fault } j_{1} \text { and a } \\
\text { nnsor fault } j_{2} \text { are isolable }
\end{gathered} \sum_{i=1}^{n}\left|f_{i j_{1}}-f_{q_{i j_{2}}}\right| \rho_{i} \geq q_{j_{2}}
$$

Note that for a non-installed sensor, the right hand side of inequality (18) becomes 0 , meaning that no isolability property is expected for this sensor fault. However, as long as a sensor is chosen for installation, equation (18) becomes equivalent to (15).

Equation (18) can be extended to any pair of system fault and sensor fault, and written in compact form as:

$$
\left[\begin{array}{ll}
\mathbf{G}_{\mathbf{2}} & -\mathbf{F}_{\mathbf{I}_{\mathbf{2}}}{ }^{T}
\end{array}\right]\left[\begin{array}{l}
\mathbf{q} \\
\rho
\end{array}\right] \leq \mathbf{0}_{l \cdot k \times 1}
$$

where

$\mathbf{F}_{\mathbf{I}_{\mathbf{2}}}=\left[f_{I_{2 i p}}\right]$ is a $n \times l \cdot k$ matrix with:

$$
f_{I_{2 i p}}=\left|f_{i j_{1}}-f_{q_{i j_{2}}}\right| \quad\left\{\begin{array}{l}
\forall j_{1} \in\{1, \ldots, l\} \\
\forall j_{2} \in\{1, \ldots, k\}
\end{array}\right.
$$

where $p$ indexes in lexicographical order the cartesian product of the system faults set and the sensor faults set. Matrix $\mathbf{G}_{\mathbf{2}}$ is used to involve the corresponding sensor in (18) according the sub-index $j_{2}$ in $p$.

$\mathbf{G}_{2}$ is the following $l \cdot k \times k$ matrix:

$$
\mathbf{G}_{\mathbf{2}}=\left[\begin{array}{llll}
I_{k} & I_{k} & \cdots & I_{k}
\end{array}\right]^{T}
$$

3) Fault isolability between sensors faults:

Now the isolability condition involves two sensor faults, so it depends on whether both sensors are considered for installation. The condition for isolability between two sensor faults can be stated as the following non-linear inequality:

$$
\sum_{i=1}^{n}\left|f_{q_{i j_{1}}}-f_{q_{i j_{2}}}\right| \rho_{i} \geq q_{j_{1}} q_{j_{2}}
$$

Note that as long as any of the two sensors are not selected for installation, the right hand side of inequality (22) becomes 0 , meaning that no isolability property is expected

\footnotetext{
${ }^{1}$ The term lexicographical order is also known as dictionary order or alphabetic order.
} 
between their corresponding sensor faults. However, as long as both sensor are selected for installation, equation (22) becomes similar to (15).

Equation (22) can be transformed into the equation in (23), which is a linear equivalent constraint.

$$
\text { two sensors faults } \leftrightarrow \sum_{i=1}^{n}\left|f_{q_{i j_{1}}}-f_{q_{i j_{2}}}\right| \rho_{i} \geq q_{j_{1}}+q_{j_{2}}-1
$$

Note that the left hand side of the equation in (23) is non-negative, so this constraint will only become meaningful when both sensors are selected for installation.

Equation (23) can be extended to any combination of two sensor faults, and written in compact form as:

$$
\left[\begin{array}{ll}
\mathbf{G}_{3} & -\mathbf{F}_{\mathbf{I}_{3}}{ }^{T}
\end{array}\right]\left[\begin{array}{l}
\mathbf{q} \\
\rho
\end{array}\right] \leq \mathbf{1}_{C_{2}^{k} \times 1}
$$

where $\mathbf{G}_{\mathbf{3}}$ is the following $C_{2}^{k} \times k$ matrix:

$$
\mathbf{G}_{3}=\left[\begin{array}{ccccc}
1 & & & \\
\vdots & & \mathbf{I}_{k-1} & \\
1 & & & \\
0 & 1 & & \\
\vdots & \vdots & \mathbf{I}_{k-2} \\
0 & 1 & & \\
& & \vdots & \\
0 & \cdots & 0 & 1 & 1
\end{array}\right]
$$

and $\mathbf{F}_{\mathbf{I}_{\mathbf{3}}}=\left[f_{I_{3 i r}}\right]$ is a $n \times C_{2}^{k}$ matrix with:

$$
f_{I_{3 i r}}=\left|f_{q_{i j_{1}}}-f_{q_{i j_{2}}}\right| \quad \forall j_{1}, j_{2} \in\{1, \ldots, k\}: j_{1}<j_{2}
$$

where $r$ indexes in lexicographical order the $C_{2}^{k}$ sensor faults combinations.

Remark that matrix $\mathbf{G}_{\mathbf{3}}$ is used to involve the corresponding pair of sensors in (23) according to the indexes $j_{1}$ and $j_{2}$ used to construct matrix $\mathbf{F}_{\mathbf{I}_{3}}$ in (26).

\section{Problem Formulation}

Once detectability and isolability constraints have been introduced, the optimal sensor placement for FDI can be formally presented. The problem is reformulated as

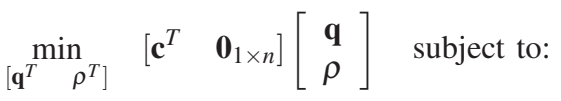

$$
\begin{aligned}
& {\left[\begin{array}{cc}
-\mathbf{M} & k \mathbf{I}_{n} \\
\mathbf{0}_{l \times k} & -\mathbf{F}^{T} \\
\mathbf{I}_{k} & -\mathbf{F}_{\mathbf{q}}{ }^{T} \\
\mathbf{0}_{C_{2}^{l} \times k} & -\mathbf{F}_{\mathbf{I}_{\mathbf{1}}}{ }^{T} \\
\mathbf{G}_{\mathbf{2}} & -\mathbf{F}_{\mathbf{I}_{\mathbf{2}}}{ }^{T} \\
\mathbf{G}_{\mathbf{3}} & -\mathbf{F}_{\mathbf{I}_{3}}{ }^{T}
\end{array}\right]\left[\begin{array}{l}
\mathbf{q} \\
\rho
\end{array}\right] \leq\left[\begin{array}{c}
\beta \\
-\mathbf{1}_{l \times 1} \\
\mathbf{0}_{k \times 1} \\
-\mathbf{1}_{C_{2}^{l} \times 1} \\
\mathbf{0}_{l \cdot k \times 1} \\
\mathbf{1}_{C_{2}^{k} \times 1}
\end{array}\right]} \\
& {\left[\begin{array}{ll}
\mathbf{q}^{T} & \rho^{T}
\end{array}\right] \text { is binary }}
\end{aligned}
$$

Constraint (28) is the concatenation of (10), (12), (14), (16), (19) and (24) respectively, where all the matrices involved have been previously defined.

The number of rows (i.e., constraints) in (28) is the following:

- The ARR selector constraints (10) involve $n$ rows.

- The detectability constraints (12) and (14) involve $l+k$ rows.

- The isolability constraints (16), (19) and (24) involve $C_{2}^{l}+l \cdot k+C_{2}^{k}=C_{2}^{l+k}$ rows.

The cost vector of the objective function is extended as a result of including $\rho$ in the variable vector. Since the goal is optimizing the set of sensors, the costs related to the $A R R$ selector, $\rho$, are set to zero. Hence, $\rho$ is regarded as a dummy vector.

In the previous development, it has been assumed that there exists a sensor configuration such that all faults concerned are fully detectable and isolable among them. Note that verifying whether this assumption is fulfilled can be accomplished by just checking which faults are detectable and isolable when all the candidate sensors are installed in the system. Should detectability and isolability of all concerned faults be non-attainable with any sensor configuration, then maximum detectability and isolability specifications should be determined. Next, the rows in (28) that involve undetectable faults or non-isolable faults pairs should be removed from the constraints set in order to make the optimization problem feasible.

\section{A. Sensor placement optimization with minimal set of ARR}

The BILP optimization stated in (27)-(29) can be extended to optimize the selected ARRs. This requires to set a cost for each ARR in the cost vector of the objective function. Therefore, the optimization problem will be expressed as follows:

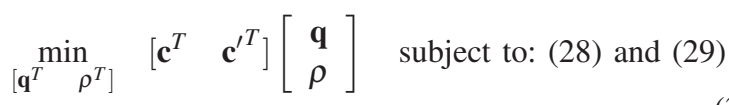

where $\mathbf{c}^{\prime}$ is a column-vector of $n$ ARR costs.

As an example of different criteria for optimizing the set of ARRs consider the following:

- Minimizing the number of the chosen ARRs: set the same cost for each ARR.

- Minimizing the complexity of the chosen ARRs: set a cost proportional to the number of equations involved in each ARR computation.

- Maximizing the isolability properties of the chosen ARRs: set a cost proportional to the number of (system and sensor) faults each ARR is sensitive to.

Usually, the main goal will be to optimize the sensors set and then, once a minimal sensor set is ensured, optimize the ARR set. Thus, given $\mathbf{c}^{\prime}=\left[\begin{array}{lll}c_{1}^{\prime} & \cdots & c_{n}^{\prime}\end{array}\right]^{T}$, condition (31) must be fulfilled.

$$
\sum_{i=1}^{n} c_{i}^{\prime}<c_{j} \quad \forall c_{j} \in \mathbf{c}, j \in\{1, \ldots, k\}
$$




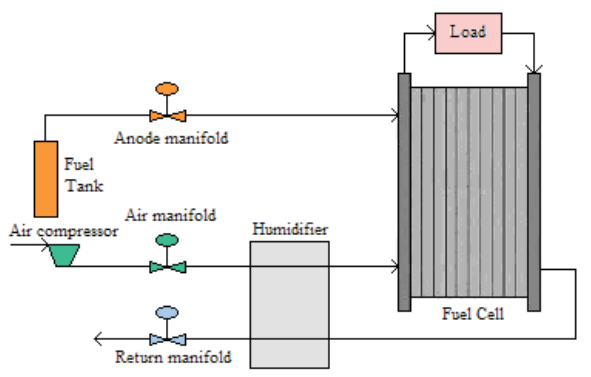

Fig. 1. Fuel Cell System

This requirement assures that the total cost of the selected ARRs will be less than the cost of choosing any sensor for installation. Thus, the ARR optimization will not affect the optimal sensor solution since the optimization algorithm will priorate the selection of a sensor configuration against the selection of the subset of selectable ARRs.

\section{Application to Fuel Cell System}

\section{A. Fuel-cell system description}

A PEM (Polymer Electrolyte Membrane) Fuel Cell System model is used to test the optimal sensor placement formulation. A model for a PEM Fuel Cell was proposed in [17]. This model is widely accepted nowadays in the control community as a good representation of the behavior of an actual fuel cell for control purposes. The main components considered in the system (Fig. 1) are the air compressor, the air manifold, the fuel cell stack, anode manifold and the return manifold. In the model, it is assumed that the temperature is known and constant, since its dynamical behaviour is much more slower than those of the rest of the model.

The structural relations between model equations and system variables are summarized below. Model equations are derived from [17] and they are classified according to the system component that they describe:

- Air Supply Compressor:

$e_{1}: f\left(\omega_{c p}, \tau_{c m}, \tau_{c p}\right)=0$

$e_{2}: f\left(\tau_{c m}, V_{c m}, \omega_{c p}\right)=0$

$e_{3}: f\left(\tau_{c p}, \omega_{c p}, p_{s m}, W_{c p}, f_{p_{s m}}\right)=0$

$e_{4}: f\left(W_{c p}, p_{s m}, \omega_{c p}, f_{p_{s m}}\right)=0$

- Air Supply Manifold:

$e_{5}: f\left(W_{\text {sm,out }}, p_{\text {sm }}, p_{c a}, f_{W_{\text {sm }, \text { out }}}\right)=0$

$e_{6}: f\left(W_{c p}, W_{\text {sm,out }}, f_{p_{s m}}, f_{W_{\text {sm }, \text { out }}}\right)=0$

- Fuel Cell Stack:

$e_{7}: f\left(W_{\text {ca,out }}, W_{v, \text { inj }}, I_{s t}, W_{\text {sm }, \text { out }}, f_{W_{\text {sm }, \text { out }}}, f_{I_{\text {st }}}, f_{n}\right)=0$

$e_{8}: f\left(W_{a n, i n}, I_{s t}, f_{I_{s t}}, f_{n}\right)=0$

$e_{9}: f\left(W_{\text {sm,out }}, p_{c a}, W_{v, \text { inj }}, I_{s t}, p_{a n}, V_{s t}, f_{W_{s m, o u t}}, f_{I_{s t}}\right)=0$

- Anode Manifold:

$e_{10}: f\left(W_{a n, i n}, p_{a n}\right)=0$

- Return Manifold:

$$
\begin{aligned}
& e_{11}: f\left(W_{\text {ca,out }}, W_{\text {rm, out }}, f_{W_{\text {rm }, \text { out }}}\right)=0 \\
& e_{12}: f\left(p_{\text {ca }}, W_{\text {ca,out }}, W_{\text {rm, out }}, f_{W_{\text {rm,out }}}\right)=0
\end{aligned}
$$

Model variables are classified into the following categories:

- Control variables: variables required for control purposes. These variables are already measured.

$V_{c m}$ : Compressor voltage

$W_{c p}$ : Air flow through the compressor

$I_{s t}:$ Stack current

$V_{s t}$ : Stack voltage

- Unmeasurable variables: variables for which sensors are not available.

$\tau_{c m}$ : Compressor motor torque

$\tau_{c p}$ : Load torque

$W_{v, \text { inj }}$ : Humidifier injector flow

- Measurable variables: define all possible sensor locations.

$\omega_{c p}$ : compressor angular speed

$p_{s m}$ : Supply manifold pressure

$W_{\text {sm,out }}$ : Supply manifold exit flow

$p_{c a}$ : Cathode pressure

$W_{\text {ca,out }}$ : Cathode output flow

$p_{a n}$ : Anode pressure

$W_{\text {an,in }}$ : Anode input flow

$W_{\text {rm,out }}$ : Return manifold exit flow

- Faults.

$f_{p_{s m}}:$ Compressor fault

$f_{W_{s m, \text { out }}}:$ Supply manifold fault

$f_{n}$ : Cell fault

$f_{I_{S t}}$ : Fuel Cell Stack fault

$f_{W_{r m, o u t}}$ : Return manifold fault

Each measurable variable has an associated sensor which together constitute the set of candidate sensors.

The FDI specifications which have to be fulfilled in this application are that system faults and installed sensors faults must be detectable and isolable.

\section{B. Optimal Sensor Placement Solution}

Given the structural model of the Fuel Cell System, and considering a fully sensored system, a total of 9039 ARRs can be found. For further information on methods devoted to finding ARRs see [16] and [8]. Then, taking into account which equation is related with each sensor or fault, matrices $M, F$ and $F_{q}$ can be extracted ${ }^{2}$.

If all candidate sensors were installed, it would be straightforward to check that all faults are detectable and isolable (assuming ARR-based exoneration): it suffices to verify that all columns in $F$ and $F_{q}$ have at least a '1', and that every possible pair of columns is different. So, an optimal sensor placement problem can be posed, since it should have at least that feasible solution.

Solving the optimal sensor placement problem requires a cost to be associated to each candidate sensor. Consider

\footnotetext{
${ }^{2}$ Due to the size of such matrices (9039 rows), they are not shown in this paper.
} 
TABLE I

SENSOR COST FOR EACH VARIABLE

\begin{tabular}{|c|c|c|c|c|c|c|c|c|}
\hline $\mathbf{x}$ & $3^{\bar{t}}$ & $\tilde{\Sigma}_{2}^{\tilde{2}}$ & 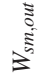 & $\Xi$ & 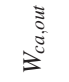 & $\Xi$ & $\stackrel{\Xi}{\Xi}$ & 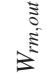 \\
\hline c1 & 400 & 600 & 500 & 300 & 1200 & 1000 & 1100 & 200 \\
\hline c2 & 500 & 100 & 200 & 700 & 300 & 600 & 900 & 400 \\
\hline
\end{tabular}

TABLE II

FSM FOR THE OPTIMAL SET OF ARRS

\begin{tabular}{|c|c|c|c|c|c|c|c|c|c|c|}
\hline & +5 & 咅 & +5 & +5 & & $\underbrace{\hat{E}}$ & $\overbrace{i=}^{\delta}$ & $\frac{E}{\sqrt{n}}$ & $\frac{\hbar}{\sum^{n}}$ & 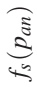 \\
\hline$A R R_{327}$ & 1 & 1 & 1 & 1 & 1 & 0 & 1 & 1 & 1 & 0 \\
\hline$A R R_{6838}$ & 1 & 1 & 0 & 0 & 1 & 1 & 1 & 0 & 0 & 0 \\
\hline$A R R_{7024}$ & 1 & 1 & 1 & 1 & 1 & 1 & 1 & 1 & 1 & 1 \\
\hline$A R R_{8735}$ & 0 & 1 & 1 & 1 & 1 & 1 & 1 & 0 & 1 & 1 \\
\hline$A R R_{8848}$ & 1 & 0 & 1 & 0 & 1 & 1 & 1 & 1 & 1 & 1 \\
\hline$A R R_{8956}$ & 1 & 1 & 0 & 1 & 0 & 1 & 1 & 1 & 1 & 1 \\
\hline
\end{tabular}

sensor cost $\mathbf{c 1}$ in Table I. Regarding the ARRs set, a cost is assigned proportional to the number of equations that each ARR relates with ( $\mathrm{nr}$. of equations $\times 10^{-3}$ ). Remark that condition (31) is fulfilled by every sensor cost, since for this example $\sum_{i=1}^{9039} c_{i}^{\prime}=82.081$.

The BILP optimization is solved and implemented using ILOG OPL Studio [18]. The result is $\mathbf{q} *=$ $\left[\begin{array}{llllllll}0 & 0 & 0 & 0 & 0 & 1 & 0 & 1\end{array}\right]^{T}$. Therefore, the optimal subset of candidate sensors must measure variables $p_{a n}$ and $W_{r m, o u t}$. Moreover, an optimal subset of ARRs is found, that depends on these sensors and guarantees fault detectability and isolability. The corresponding Fault Signature Matrix is shown in Table II. Remark, in the table, that $f_{s}(z)$ stands for a fault in the sensor measuring variable $z$.

Changing the ARR cost assignment criterium a different solution is obtained. Assigning now a cost proportional to the number of faults each ARR is sensitive to ( $\mathrm{nr}$. of faults $\times 10^{-3}$ ), a new set of ARRs is chosen $\left(\left\{A R R_{6838}, A R R_{8736}, A R R_{8914}, A R R_{8970}, A R R_{8982}, A R R_{8988}\right\}\right)$, such that faults are detectable and isolable.

On the other hand, changing the sensor cost assignment, a different set of sensors and ARRs is obtained. Consider now the sensor cost $\mathbf{c} \mathbf{2}$ defined in Table I, and an ARR cost proportional to the number of faults each ARR is sensitive to ( $\mathrm{n}$. of faults $\times 10^{-3}$ ). Applying the BILP formulation and solving the optimization problem, the optimal set of candidate sensors found must now measure variables $W_{c a, o u t}$ and $p_{a n}$. And the corresponding optimal subset of ARRs is $\left\{A R R_{6839}, A R R_{7941}, A R R_{8738}, A R R_{8850}, A R R_{8970}, A R R_{8990}\right\}$

\section{Conclusions}

In this work, a new methodology to solve the sensor placement problem for FDI has been addressed and applied to a Fuel Cell System. The sensor placement problem has been presented formally as a binary variable problem. The novelty is that BILP standard formulation is used, therefore standard algorithms to solve BILP optimization can be used. The advantage is that these algorithms are deeply developed and their branch and bound search is well-studied, leading to a fast resolution in the majority of cases.

The main drawback of the presented approach is that the ARRs set must be provided beforehand. It is known that generating the whole set of ARRs is a computational complex task. Future works might be improved by solving the BILP optimization without the need of generating such ARRs set.

The authors are aware that the ARR-based exoneration assumption may be unrealistic in many cases. However, this method could be easily extended to the case when a residual does not always cross the threshold at fault occurrence.

\section{REFERENCES}

[1] M. Staroswiecki and G. Comtet-Varga, "Analytical redundancy relations for fault detection and isolation in algebraic dynamic systems," Automatica, vol. 37, no. 5, pp. 687-699, 2001.

[2] R. Nikoukhah, "A new methodology for observer design and implementation," IEEE Trans. Automat. Contr., vol. 43, no. 2, pp. 229-234, 1998

[3] D. Maquin, M. Luong, and J. Ragot, "Fault detection and isolation and sensor network design," Europ. J. Autom., vol. 31, no. 13, pp. 396-406, 1997.

[4] R. Raghuraj, M. Bhushan, and R. Rengaswamy, "Locating sensors in complex chemical plants based on fault diagnostic observability criteria," AIChE J., vol. 45, no. 2, pp. 310-322, Feb. 1999.

[5] M. Krysander and E. Frisk, "Sensor placement for fault diagnosis," IEEE Trans. Syst., Man, Cybern. A, vol. 38, no. 6, pp. 1398-1410, 2008.

[6] C. Commault, J. M. Dion, and S. Y. Agha, "Structural analysis for the sensor location problem in fault detection and isolation," Automatica, vol. 44, no. 8, pp. 2074-2080, aug 2008.

[7] A. A. Yassine, S. Ploix, and J. M. Flaus, "A method for sensor placement taking into account diagnosability criteria," Int. J. Appl. Math. Comput. Sci., vol. 18, no. 4, pp. 497-512, 2008.

[8] L. Travé-Massuyès, T. Escobet, and X. Olive, "Diagnosability analysis based on component supported analytical redundancy relations," IEEE Trans. Syst., Man, Cybern. A, vol. 36, no. 6, pp. 1146-1160, 2006.

[9] A. Chamseddine, H. Noura, T. Raharijaona, and M. Ouldsine, "Structural analysis-based sensor location for diagnosis as optimization problem," in Proc. 46th IEEE Conference on Decision and Control, New Orleans, USA, Dec. 12-14, 2007, pp. 6154-6159.

[10] A. Rosich, R. Sarrate, V. Puig, and T. Escobet, "Efficient optimal sensor placement for model-based FDI using and incremental algorithm," in Proc. 46th IEEE Conference on Decision and Control, New Orleans, USA, Dec. 12-14, 2007, pp. 2590-2595.

[11] M. Blanke, M. Kinnaert, J. Lunze, and M. Staroswiecki, Diagnosis and Fault-Tolerant Control, 2nd ed. Springer, 2006.

[12] R. Sarrate, V. Puig, T. Escobet, and A. Rosich, "Optimal sensor placement for model-based fault detection and isolation," in Proc. 46th IEEE Conference on Decision and Control, New Orleans, USA, Dec. 12-14, 2007, pp. 2584-2589.

[13] L. A. Wosley, Integer Programming. New York, USA: John Wiley \& Sons, 1998

[14] J.-M. Dion, C. Commault, and J. van der Woude, "Generic properties and control of linear strcutured systems: a survey," Automatica, vol. 39, pp. 1125-1144, 2003.

[15] A. Rosich, R. Sarrate, and F. Nejjari, "Optimal sensor placement for FDI using binary integer linear programing," in Proc. DX'09, 20th International Workshop on Principles of Diagnosis, Stockholm, 14 $17,2009$.

[16] M. Krysander, J. Åslund, and M. Nyberg, "An efficient algorithm for finding minimal over-constrained sub-systems for model-based diagnosis," IEEE Trans. Syst., Man, Cybern. A, vol. 38, no. 1, 2008.

[17] J. T. Pukrushpan, H. P., and A. G. Stefanopoulou, "Analysis for automotive fuel cell systems," Transactions of the ASME, vol. 126, pp. 14-25, 2004.

[18] The ILOG website. [Online]. Available: http://www.ilog.com/ 